\title{
Presupposition ad Entailment
}

\author{
Yuanli Fan \\ School of Foreign Studies, Xi'an University, Shaanxi, Xi'an, 710065)
}

Key words: Presupposition; Entailment; Negation test; Information focus

\begin{abstract}
This paper aims at the distinction of Presupposition and Entailment. Since various kinds of definition misused confusedly. The distinction is always a hot question for discussing. The author tries to distinguish the presupposition and entailment from semantic scope by negation test. The traditional negation test seems to have its limitation for the semantic negation has its function scope. Therefore, the information focus is introduced to make a distinction between semantic presupposition and entailment of a multi-elements sentence. Through analyzing preposition and entailment of English sentence, the semantic and pragmatic essence can be easily acquired in order to promote the English Communication Competence. However, some sentence pattern, such as the Imperative sentence, is needed to do further research.
\end{abstract}

\section{Introduction}

Entailment and presupposition shows the different relationship between sentences. Both of them are the meaning and information deduced from the sentence itself. The distinction between the two is always a attentive hot question and has not reached agreement. The key point about that lie in: firstly, different referring terms are used by the same symbol and causing the misunderstanding about presupposition and entailment. It is essential to make clear distinction of presupposition and entailment for the developing of pragmatic or semantic research.In this paper, we will discuss the semantic presupposition, and the distinction of semantic presupposition and entailment.

Presupposition originates with debates in philosophy, specifically debates about the nature of reference and referring expressions. Such problems lie at the heart of logical theory and arise from consideration of how referring expressions in natural language should be translated into the restricted logical languages. Frege, the first philosopher in recent times is the architect of modern logic. He raised many of the issues that were later to become central to discussions of presupposition. He said:

If anything is asserted there is always obvious presupposition that the simple of compound proper names used have a reference. If one therefore asserts 'Kepler died in misery.'there is a presupposition that the name Kepler designates something.( Frege,1892)

Although the assertion of the sentence 'Kepler died in misery.(A)'does not include 'Kepler existed. (B)', it is obviously that sentence B presuppose sentence B.

Meanwhile he pointed out that sentence "Kepler didn’t die in misery." and the sentence "Kepler died in misery.” shares the same presupposition, i.e. 'Kepler existed. (B)'

Frege thus sketches a theory of presupposition with the following propositions:

i, Referring phrases and temporal clauses (for example) carry presuppositions to the effect that they do in fact refer.

ii, A sentence and its negative counterpart share the same set of presuppositions

iii, In order for an assertion of a sentence to be either true of false, its presuppositions must be true or satisfied. 
In addition presupposition was a focus area in linguistic theory during the period 1969-1976, because it raised substantial problems for almost all kinds of linguistic theories then available. When Strawson's notion of presupposition came to the attention of linguists, it seemed to open up a new and interesting possibility. Up to this point linguists had been operating with one crucial semantic relation in particular, namely entailment. This relation can be defined in terms of in valid rules of inference or alternatively in terms of the assignment of truth and falsity. Semantic entailment is thus definable as follows:

A semantically entails B iff every situation that makes A true, makes B true(or in all words in which $\mathrm{A}$ is true $\mathrm{B}$ is true)

Eg: that person is a bachelor.(A)

This sentence entails the sentence below:

That person is a man.(B)

In whatever conditions, if someone is a bachelor, he must be a man. However if sentence A is not true, what about its entailment? If we say: that person is not a bachelor. Then the entailment B is hard to confirm. Since if one is not a bachelor, he can be a man and she can be a woman. Therefore, if sentence $A$ entails sentence $B$, sentence A must satisfy two conditions:

1) if $A$ is true, then $B$ is true

2) if $A$ is not true, then $B$ can be true or not true.

Such a relation is basic to semantics. Not only does it capture logical truths, but all the other essential semantic relation can be directly defined in terms of it. The interesting possibility opened up by the notion of presupposition was that we might be able to add a new and distinct semantic relation to the inventory of the well-known ones. In doing so we would be bringing logical models more into line with natural language semantics. This programme, the creation of a new, well defined semantic relation that would play a role within a number of theories of semantic presupposition.

The semantic presupposition is inference for the proposition of one sentence. If $\mathrm{A}$ and $\mathrm{B}$ constitutes the presupposition relation, the following two condition must be satisfied:

1) if $\mathrm{A}$ is true, $\mathrm{B}$ is true too.

2) If $A$ is not true, but $B$ is true.

E.g. John is married.

The presupposition is: John exists.

If it is not true, ie John is not married, the presupposition 'John exists' is also true.

It is clear that the distinction of entailment and presupposition lies in the second condition. Namely, when sentence A is not true or sentence A is denied, the inference can not be existed. According to the difference the linguists create the "Negation Test"to estimate the inference for a sentence, entailment or presupposition.

For example: John managed to stop the car (1)

We can infer the following two sentences at least:

John stopped the car.

John tried to stop the car.

The negative of the sentence 1 is : John did not manage to stop the car. (4)

It is obviously that from sentence 4 we can not infer sentence 2, however from sentence 1 and 4 we can infer sentence 3 . Therefore sentence 2 is the entailment of sentence 1 , written sentence 1 $\rightarrow$ sentence 2 ; and sentence 3 is the presupposition of sentence 1 , written as sentence $1>$ sentence 3. 
If according to "negation test" strictly, how about the presupposition of the interrogative sentence, the imperative sentence, and the exclamatory sentence?

For example:(5)Do you stop beating your wife?

There is a negation scope and negative semanicreferrence when the sentence is formed with many components. Take (5) as example: the possible answer to it is:

(5) a, Yes, I stop doing that.

b, No, I don't stop doing that.

The (1) a, and b, presuppose the fact that speaker did beat his wife before. For the questions we should bear in mind that they share the same presuppostion as their answer. And of course, the answer “( 1 c, $)$ I haven't doing that” is reasonable in real context. However, that beyond the semantic presupposition.

If the sentence is formed with too many meaningful element, the negation scope and the negative referring meaning should pay attention to. How to deny the sentence? Which part of the sentence is denied?Because sometimes both of them can be denied.

\section{Introduction of Entailment}

From 2.4 we can conclude that the“negation test”can not solve all the problems, sometimes there are the phenomenon of invalidation of it. For example:

A

(6)a, the Chinese eat with chopsticks.

b, the Chinese doesn't eat with chopsticks.

We have discussed thata sentence and its negative counterpart share the same set of presuppositions. Here, it seems that $b$ is the presupposition of $a$. However, only in sentence $b$, the $b$ is the presupposition of a. In every sentence $a, b$ is the entailment of $a$. our question is: why in the positive sentence $\mathrm{B}$ is the entailment not the presupposition? There are two points which can help us understand it:

Firstly, B is similar with the assertion of A.Shi Anshiexplained this point clearly, he explained entailment is the contents which assertion of utterance shows; and presupposition is beyond that. Secondly, the entailment of a sentence is subordinate to the original sentence or the entailment is a part of the originate sentence. Shi Anshi said that all the entailments are belong to the basic information of the utterance. And the basic information is the contents which the speaker discusses. For example:

(7) That person is a bachelor.

That person is a man.

In the example of ( 7 ), the basic information is someone is something. We can see that bachelor and man, is a kind of relation of a part and the whole. From this we can conclude that the assertion is the affirmation of the reality of objective, the generalization of a sentence's basic information.

Chen ping stated that there is an universal phenomenon in the negative sentence in Chinese and English. Although all the predicate is include in negative words, factually the negative words always deny one or several elements of a sentence, not deny all elements of it.

In one sentence the adverbial modifier, the complement, the object could be the denying objects if they are included in the negative range and meanwhile the predicate part retain positive. Then the denied element becomes the focus information, shows the contents of the assertion of the sentence, and the information beyond the assertion is the presupposition of the sentence.

For example:"He didn't clean the table thoroughly" In this sentence, the complement of the sentence "clean the table thoroughly" is the object which the negative words deny. so "the table is 
not very clean" is the focus information of the sentence. Therefore "he have cleaned the table"is a presupposition and "the table is not very clean" is a entailment.

We can conclude a definition of entailment and presupposition basing the focus information.

Presupposition is trigger by the sentence, and basing on which the speaker wishes the listener understand the given information as he anticipates.

Entailmentis the conclusion which the listener infers form the speaker's utterance,that is new information of a sentence.

And the distinctive characteristics of presupposition and characteristic can be concluded as follows:

Firstly, the essential distinct between the two is the focus information. The presupposition information has nothing to do with the focus information, whereas entailment can reflect the focus information or not. That is to say, presupposition doesn't reflect the focus information at all, and entailment can reflect any part information of the sentence.

For example:

Sentence:A married B.

Presupposition: There are two person named A and B.

Entailment: a, There are two person named A and B.

b,A man and a woman got married.

c,A and b, they love each other and eventually become a couple.

Although entailment doesn't involve the focus information, generally speaking, the meaningful entailment reflects the focus information inevitably just as Entailment b and c. whereas Entailment a, is meaningless unless there is a particular communication surroundings. Since information communication can not always talks about the inexisted people or things. Whereas presupposition must make a clear statement of time, place, people and relative incidents to the speaker and in order to make the communication successfully.

Secondly, proposition is true, incident is true, so the entailment is true; proposition is false, incident is false, the entailment is meaningless (can be true or false).whereas presupposition is related closely with the proposition and has nothing to do with the fact the proposition conceals. Therefore, no matter the proposition is true or false, the presupposition is always true. It is true that the presupposition sometimes reflects falsity but the speaker and the listener suppose it is true.

Thirdly, presupposition is the semantic information which the speaker hopes the listener could understand it as the speaker anticipates. The semantic information of entailment is inferred from the sentence itself not depends on context and keep unchanged while the context changes.

From the analysis we can see that: in a sentence, a part belongs to the focus, and the left part belongs to presupposition. Yuan Shulin ( 2000 ) said that focus structrution determine the presupposition meaning and the focus effect presuppostion from the level of a sentence.

\section{Conclusion}

Presupposition has been an important topic in pragmatics and we can see lively debates in the literature such as the distinctions between presupposition and entailment, between presupposition and implicature etc. From this paper we can see that in semantic presupposition the presupposition and entailment is distinguished. The negation test is not omnipotent and the focus, given information, new information is introduced to distinguish semantic presupposition and entailment. However, some sentence pattern such as the imperative sentence, and the exclamatory sentence is need to do a further research. 


\section{Reference}

[1] Levison, S.C. (2001). Pragmatics. Shanghai: Foreigh Language Teaching and Research Press

[2] Chen Ping, (1996) .Comparative Study on the Negative Structure Between English and Chinese.Shanghai: Foreigh Language Education Press

[3] He Zhaoxiong, (2000).A New Introduction to Pragmatics.Shanghai: Foreigh Language Education Press

[4] He Ziran, (1997) .PRAGMATICS AND ENGLISH LEARNING.Shanghai: Foreigh Language Education Press

[5] Li Ruihua,(1996).Language and Culture: Contrastive Studies Between English and Chinese.Shanghai: Foreigh Language Education Press

[6] Shi Anshi,(1994).A Study on Semantic. Beijing:BeijingLanguage and Literature Press

[7] XuLiejiong\&Pan Haihua,(2005).Shanghai: Foreigh Language Teaching and Research Press

[8] Yuan Lirong, (2003) .The Limitation of the Method of "Negation Test" of Presupposition Journal of YIBinShool,2, 56-61.

[9] Yang Nianbao, (2005). Semantic Presupposition and PragmaticPresuppositionJournal of Yunmeng,3, 33-39

[10] Guocai Zeng,(2010).The Presupposition and Entailment in English Reading Comprehensition Test, Journal of Changsha University. Vol.24 NO.3 